\title{
Desenvolvimento da metodologia para síntese do poli(ácido lático-co-ácido glicólico) para utilização na produção de fontes radioativas
}

\section{Development of methodology for the synthesis of poly(lactic acid-co-glycolic acid) for use in the production of radioactive sources}

\author{
Fernando dos Santos Peleias Junior ${ }^{1,2^{*}}$, Carlos Alberto Zeituni', Maria Elisa Chuery Martins Rostelato', \\ Guilhermino José Macêdo Fechine ${ }^{3}$, Carla Daruich de Souza ${ }^{1}$, Fábio Rodrigues de Mattos ${ }^{1}$, \\ Eduardo Santana de Moura1', João Augusto Moura1', Marcos Antônio Gimenes Benega', \\ Anselmo Feher ${ }^{1}$, Osvaldo Luiz da Costa ${ }^{1}$ e Bruna Teiga Rodrigues ${ }^{1}$ \\ ${ }^{1}$ Centro de Tecnologia das Radiações - CTR, Instituto de Pesquisas Energéticas e Nucleares - IPEN, \\ São Paulo, SP, Brasil \\ ${ }^{2}$ Department of Chemistry, Centre for Sustainable Chemical Technologies, University of Bath, \\ Bath BA2 7AY, Bath, Somerset, United Kingdom \\ ${ }^{3}$ Escola de Engenharia, Universidade Presbiteriana Mackenzie, São Paulo, SP, Brasil \\ *fernandopeleias@gmail.com
}

\begin{abstract}
Resumo
A Organização Mundial da Saúde (OMS) relata o câncer como uma das principais causas de morte no mundo. Uma modalidade de tratamento que vem sendo bastante utilizada no tratamento do câncer de próstata é a braquiterapia, que consiste na introdução de sementes com material radioativo no interior do orgão. Sementes de Iodo-125 podem ser inseridas soltas ou em cordas poliméricas fabricadas a partir do (poli(ácido lático-co-ácido glicólico)) (PLGA). Foi proposto neste trabalho, o estudo e desenvolvimento da metodologia de síntese do biopolímero PLGA. Os resultados obtidos demonstram que, através da metodologia utilizada, foi possível determinar os melhores parâmetros de reação (tempo e temperatura) para o PLGA na proporção 80/20 (lactídeo/glicolídeo). Com uma temperatura de $110^{\circ} \mathrm{C}$ e tempo de reação $72 \mathrm{~h}$ o rendimento da reação é superior a $90 \%$. Os valores de massas moleculares obtidas entre os testes, ainda são baixos quando comparados com os valores obtidos por outros autores na literatura. Novos testes estão sendo conduzidos, utilizando dímeros preparados no laboratório. Testes substituindo o vácuo por uma atmosfera de nitrogênio também estão sendo realizados. Essas duas substituições podem aumentar o valor final da massa molecular do polímero. Em relação à caracterização, as técnicas utilizadas confirmaram a estrutura esperada do polímero.
\end{abstract}

Palavras-chave: câncer de próstata, braquiterapia, poli(ácido lático-co-ácido glicólico), PLGA.

\begin{abstract}
According to the World Health Organization, cancer is a leading cause of death worldwide. A radiotherapy method extensively used in prostate cancer is brachytherapy, where the area requiring treatment receives radioactive seeds. Iodine-125 seeds can be inserted loose or stranded in bioabsorbable polymers produced from poly(lactic-co-glycolic acid) (PLGA). We developed the synthesis methodology for PLGA and the results obtained show that it was possible to determine the optimal reaction parameters (time and temperature) for PLGA in 80/20 (lactide/glycolide) ratio. The yield was higher than $90 \%$ using a temperature of $110^{\circ} \mathrm{C}$ and reaction time of 72 hours; however, the molecular weight values obtained are very low compared to those obtained by other authors. New tests using previously synthesized dimers and nitrogen atmosphere are being performed. These conditions could potentially increase the molar mass of PLGA. All techniques used confirmed the expected structure of the polymer.
\end{abstract}

Keywords: prostate cancer, brachytherapy, poly(lactic-co-glycolic acid), PLGA. 


\section{Introdução}

A Organização Mundial da Saúde (OMS) relata o câncer como uma das principais causas de morte no mundo ${ }^{[1-3]}$. O câncer de próstata é o segundo tipo de câncer mais prevalente em homens ${ }^{[4,5]}$. Com o crescimento da expectativa de vida mundial, é esperado que o número de casos novos aumente cerca de $60 \%$ até o ano de 2015 . Uma modalidade que vem sendo bastante utilizada com sucesso é a braquiterapia, que consiste na introdução de sementes de Iodo-125 radioativo no interior da próstata, próximas ao tumor, afetando ao mínimo outros órgãos nas proximidades ${ }^{[6,7]}$. No cenário brasileiro, a maior parte das sementes de Iodo- 125 utilizadas são recobertas por um material polimérico bioabsorvível (poli(ácido lactico-co-glicólico)-PLGA). A função do PLGA é facilitar a fixação das sementes na próstata, reduzindo a ocorrência de migrações de sementes para outros órgãos ${ }^{[8]}$. O Instituto de Pesquisas Energéticas e Nucleares (IPEN) estabeleceu um projeto para fabricação de sementes de Iodo-125 e, devido às exigências do mercado nacional, surgiu a necessidade de produção de PLGA para a cobertura das sementes.

\subsection{Métodos para síntese de PLGA}

Os processos de polimerização dos poli( $\alpha$-hidroxiácidos carboxílicos) necessitam que os monômeros utilizados tenham alto teor de pureza, uma vez que as impurezas interferem nos caminhos da reação gerando polímeros de baixa qualidade. Dentre essas impurezas destacam-se a água e compostos contendo os grupos funcionais hidroxila e carboxila, que podem reduzir a massa molecular final e a taxa de polimerização, respectivamente ${ }^{[9]}$. Existem quatro métodos possíveis para produção dos poli $(\alpha$-hidroxiácidos carboxílicos): polimerização por policondensação direta, policondensação azeotrópica, polimerização em estado sólido (SSP), e polimerização por abertura de anéis $(\mathrm{ROP})^{[9,10]}$.

Nos dois primeiros métodos há eliminação de moléculas de água e, de um modo geral, o polímero final obtido possui baixa massa molecular. Embora a policondensação azeotrópica permita a obtenção de massas moleculares maiores, existe o problema da toxicidade do solvente associada ${ }^{[9,11]}$. Através da polimerização no estado sólido, também é possível preparar polímeros com massas moleculares elevadas. No entanto, o método exige altos tempos de reação, e também há necessidade de um pré-polímero de baixa massa molecular ${ }^{[9,12,13]}$. Por último, a polimerização por abertura de anel se destaca como método preferido para produção de PLGA. Neste tipo de polimerização, parte-se dos dímeros cíclicos lactídeo e glicolídeo. Através da abertura dos anéis dos dímeros, tem-se a geração de uma bifuncionalidade, que ao reagir com outras unidades monoméricas, forma uma cadeia polimérica. Neste tipo de polimerização não há a formação de subprodutos durante a reação. Os dímeros cíclicos são produzidos a partir da despolimerização de polímeros de baixa massa molecular obtidos por policondensação ${ }^{[9,11,14,15]}$.

A síntese de polímeros por abertura de anel é o método mais comumente estudado devido a possibilidade de variar as propriedades dos polímeros resultantes de forma mais controlada, ampliando o seu campo de aplicação. Como os dímeros lactídeo e glicolídeo podem apresentar impurezas, como a água, ou até mesmo os monômeros que não reagiram, é de extrema importância que um processo cuidadoso de purificação desses dímeros seja realizado ${ }^{[9]}$.

O mecanismo mais utilizado para esse tipo de polimerização é o de coordenação-inserção, pois permite a obtenção de materiais com alta massa molecular ${ }^{[9]}$. A Figura 1 ilustra o mecanismo de coordenação inserção, através da polimerização do lactídeo, utilizando alcóxido de alumínio como catalisador.

$\mathrm{O}$ primeiro passo do mecanismo ocorre quando o oxigênio exocíclico do dímero forma temporarioamente um composto de coordenação com o metal presente no catalisador. Essa ligação aumenta a nucleofilicidade do catalisador (no exemplo acima, o alcóxido de alumínio), assim como a eletrofilicidade do grupo carbonila presente no dímero. No segundo passo, a ligação acila - oxigênio é quebrada e o lactídeo já aberto, é inserido no ligação metal-oxigênio do catalisador ${ }^{[9,16,17]}$. A reação continua conforme os anéis

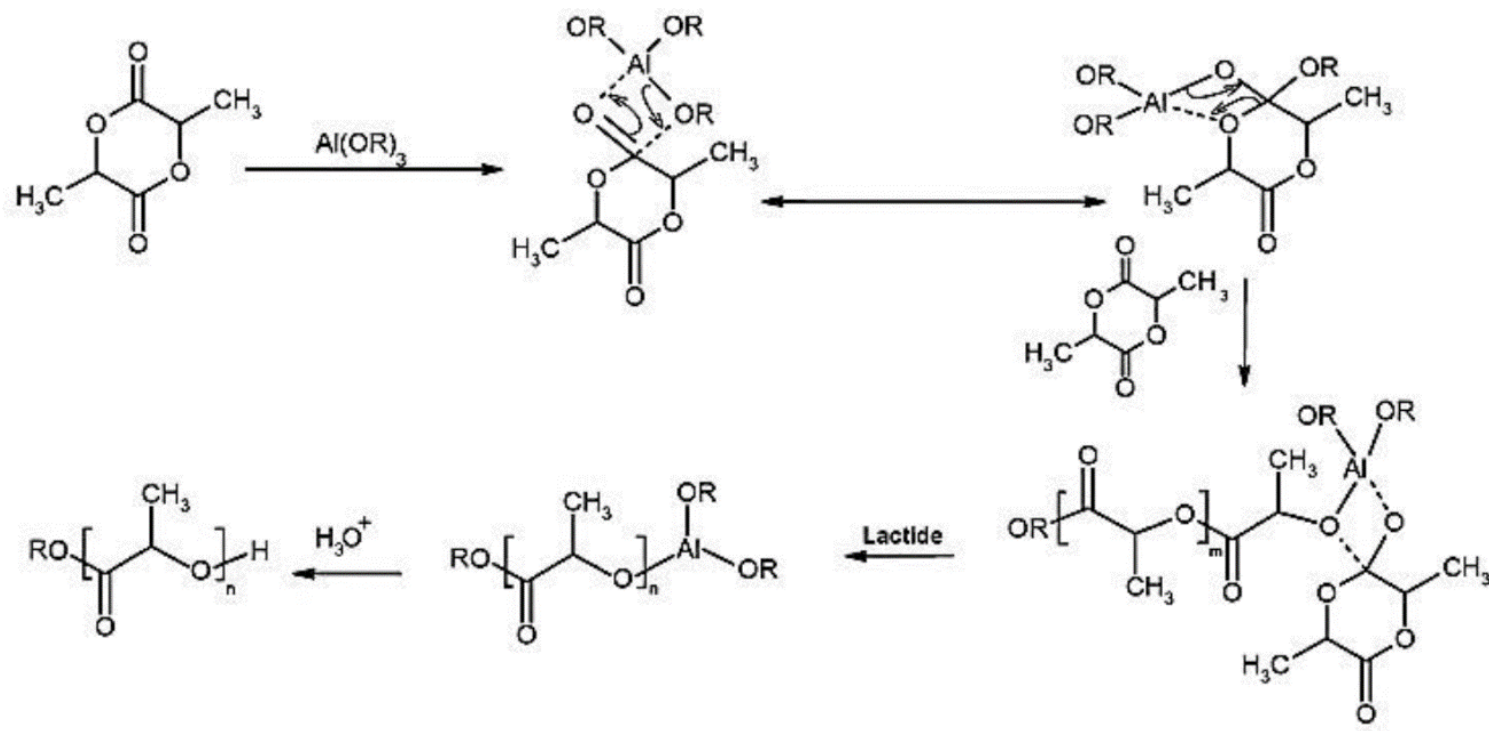

Figura 1. Demonstração do mecanismo de coordenação-inserção na polimerização em PLA ${ }^{[9]}$. 
de outras moléculas de lactídeo são abertos e inseridos no catalisador entre o átomo do metal e oxigênio adjacente ${ }^{[16,17]}$.

Variando-se condições da reação (tempo, temperatura e concentração do catalisador), polímeros de diferentes massas moleculares são obtidos. O mecanismo de reação do glicolídeo é o mesmo, pois a única diferença é a ausência dos radicais metila. Embora uma grande variedade de catalisadores tenha sido pesquisada, o 2-etilhexanoato de estanho (octanoato de estanho - $\mathrm{Sn}(\mathrm{Oct})_{2}$ é o mais utilizado em aplicações biomédicas devido a sua alta eficiência $\mathrm{e}$ baixa toxicidade ${ }^{[9,18-20]}$.

As vantagens da polimerização por abertura de anel são: menor tempo de reação e maiores taxas de conversão do monômero. Como desvantagens destacam-se: custos mais altos de produção e dificuldades técnicas para obtenção dos dímeros cíclicos com elevada pureza ${ }^{[18,19]}$.

O objetivo deste trabalho é desenvolver uma possível metodologia de síntese PLGA, utilizando o método de polimerização por abertura de anéis, com o propósito de utilizar o material sintetizado para recobrir as sementes de Iodo-125 fabricadas no laboratório do IPEN.

\section{Experimental}

Nas reações propostas neste trabalho, foram utilizados os dímeros L-lactídeo e glicolídeo, já purificados, na proporção de 80:20 respectivamente. O motivo da escolha desta proporção se deu pelo fato do glicolide ser extremamente insolúvel em quase todos os solventes orgânicos, com exceção dos solventes fluorados. No entanto, a utilização de destes solventes tornaria o projeto inviável, uma vez que $100 \mathrm{~mL}$ de hexafluoroisopropanol na empresa Sigma-Aldrich, tem um custo aproximado de R \$2000,00. Não sendo possível sintetizar um produto similar ao Vycril ${ }^{\circledR}(90 \%$ Glicolídeo/ 10\% Lactídeo), que já é utilizado em braquiterapia prostática, optou-se pela utilização da proporção descrita acima, que também é utilizada na fabricação de suturas para fixação de tendões e ligamentos aos ossos ${ }^{[21,22]}$.

Uma vez determinada a proporção dos monômeros desejada, determinou-se quais parâmetros seriam avaliados nas reações. Considerando que a reação será conduzida em bulk, ou seja, sem adição de solventes, os principais parâmetros a serem avaliados seriam: tempo, temperatura e concentração do catalisador. Neste trabalho optou-se por avaliar apenas a influência da temperatura e do tempo de reação, pois a concentração do catalisador $\left(\mathrm{SnOct}_{2}\right)$ de 1/5000 é unânime em todas as referências consultadas. Em relação ao tempo de reação e temperatura, existe uma grande faixa de valores utilizados, que varia de autor para autor.

\subsection{Síntese do poli(ácido lático-co-ácido glicólico)}

Os dímeros L-lactídeo e glicolídeo, e o catalisador octanoato de estanho ( SnOct $_{2}$ ), utilizados nas reações, foram adquiridos da empresa Sigma-Aldrich ${ }^{\circledR}$. As massas correspondentes dos dímeros e do catalisador foram adicionadas a uma ampola de vidro, que foi posteriormente selada a vácuo, a fim de que a reação se desse na ausência de $\mathrm{O}_{2}$ e possível umidade contida no ar.

As ampolas foram imersas em um banho termostático da marca Lauda, modelo E200. Foram escolhidas 3 faixas de temperatura $\left(110{ }^{\circ} \mathrm{C}, 140{ }^{\circ} \mathrm{C}, 170{ }^{\circ} \mathrm{C}\right)$ e 4 diferentes tempos de reação (6h, 24h, 72h, 168h). Esses valores foram escolhidos baseados nos diferentes parâmetros encontrados nas referências estudadas ${ }^{[18,20,23-25]}$. Cada teste foi realizado em duplicata, totalizando 24 reações. As ampolas foram numeradas de 1 a 24 . A Tabela 1 relaciona o número de cada ampola com os parâmetros utilizados, assim como as massas dos monômeros e catalisador pesadas.

Decorrido o tempo proposto para cada reação, as ampolas foram retiradas do banho e mergulhadas em banho

Tabela 1. Valores de massa e parâmetros utilizados nas reações de polimerização.

\begin{tabular}{|c|c|c|c|c|c|c|c|}
\hline $\mathrm{N}^{0}$ da Ampola & $\begin{array}{c}\text { Massa de } \\
\text { L-Lactídeo (g) }\end{array}$ & $\begin{array}{c}\text { \% em mol de } \\
\text { L-lactídeo }\end{array}$ & $\begin{array}{c}\text { Massa de } \\
\text { glicolídeo (g) }\end{array}$ & $\begin{array}{c}\text { \% em mol de } \\
\text { glicolídeo }\end{array}$ & $\begin{array}{c}\text { Massa de } \\
\text { SnOct }_{2}\end{array}$ & Tempo (h) & $\begin{array}{c}\text { Temperatura } \\
\left({ }^{\circ} \mathrm{C}\right)\end{array}$ \\
\hline 1 & 5,973 & 79,91 & 1,209 & 20,09 & 0,0052 & 6 & 110 \\
\hline 2 & 5,972 & 79,92 & 1,208 & 20,08 & 0,0048 & 6 & 110 \\
\hline 3 & 5,973 & 79,93 & 1,208 & 20,07 & 0,0054 & 24 & 110 \\
\hline 4 & 5,971 & 79,99 & 1,203 & 20,01 & 0,0053 & 24 & 110 \\
\hline 5 & 5,982 & 79,92 & 1,210 & 20,08 & 0,0048 & 72 & 110 \\
\hline 6 & 5,979 & 80,00 & 1,204 & 20,00 & 0,0049 & 72 & 110 \\
\hline 7 & 5,974 & 79,89 & 1,211 & 20,11 & 0,0056 & 168 & 110 \\
\hline 8 & 5,977 & 79,92 & 1,209 & 20,08 & 0,0053 & 168 & 110 \\
\hline 9 & 5,970 & 79,93 & 1,207 & 20,07 & 0,0061 & 6 & 140 \\
\hline 10 & 5,973 & 79,97 & 1,205 & 20,03 & 0,0052 & 6 & 140 \\
\hline 11 & 5,972 & 79,99 & 1,203 & 20,01 & 0,0049 & 24 & 140 \\
\hline 12 & 5,979 & 80,01 & 1,203 & 19,99 & 0,0052 & 24 & 140 \\
\hline 13 & 5,976 & 79,96 & 1,206 & 20,04 & 0,0049 & 72 & 140 \\
\hline 14 & 5,973 & 79,90 & 1,210 & 20,10 & 0,0052 & 72 & 140 \\
\hline 15 & 5,980 & 80,01 & 1,203 & 19,99 & 0,0053 & 168 & 140 \\
\hline 16 & 5,979 & 80,01 & 1,203 & 19,99 & 0,0050 & 168 & 140 \\
\hline 17 & 5,970 & 79,96 & 1,205 & 20,04 & 0,0042 & 6 & 170 \\
\hline 18 & 5,970 & 80,00 & 1,202 & 20,00 & 0,0046 & 6 & 170 \\
\hline 19 & 5,971 & 79,98 & 1,204 & 20,02 & 0,0039 & 24 & 170 \\
\hline 20 & 5,970 & 80,00 & 1,202 & 20,00 & 0,0043 & 24 & 170 \\
\hline 21 & 5,971 & 79,98 & 1,204 & 20,02 & 0,0052 & 72 & 170 \\
\hline 22 & 5,971 & 79,98 & 1,204 & 20,02 & 0,0051 & 72 & 170 \\
\hline 23 & 5,971 & 80,00 & 1,202 & 20,00 & 0,0055 & 168 & 170 \\
\hline 24 & 5,972 & 79,99 & 1,203 & 20,01 & 0,0050 & 168 & 170 \\
\hline
\end{tabular}


de água com gelo por 15 minutos, de modo que a reação fosse interrompida. A seguir a ampola foi quebrada, e o polímero resultante foi dissolvido, sob agitação constante, em $50 \mathrm{~mL}$ de clorofórmio $\left(\right.$ Synthlab ${ }^{\circledR}$ ). O tempo para dissolução completa do polímero variou de 1 a 12 horas, dependendo dos parâmetros da reação. Finalizada a dissolução, o polímero foi precipitado através do gotejamento de metanol $\left(\right.$ Synthlab $\left.^{\circledR}\right)$, na solução que continha o polímero dissolvido. O metanol deve ser adicionado até o ponto em que a solução polímero + clorofórmio deixe de tornar-se turva com o gotejamento.

Após a precipitação, o polímero obtido foi submetido à filtração à vácuo. Foi utilizado papel de filtro grau 589/3, que possui velocidade de filtração lenta, mas é o mais eficiente na coleta de partículas pequenas $(<2 \mu \mathrm{m})$. Logo após a filtração do polímero, o material foi submetido ao processo de secagem, sendo primeiramente levado a uma estufa por 1 hora a uma temperatura de $80^{\circ} \mathrm{C}$. Posteriormente, o polímero foi levado a um dessecador à vácuo por 24 horas, de modo que qualquer resíduo de solvente ainda presente fosse eliminado. Finalmente, o material seco foi pesado e o rendimento pode ser calculado.

\subsection{Determinação da massa molecular por cromatografia de permeação em gel (GPC)}

Para a obtenção dos dados referentes ás massas moleculares das 24 amostras de polímeros produzidos, utilizou-se um cromatógrafo HT-GPC-module 350A da marca Viscotek, equipado com colunas GPC HT-806M da marca Shodex. Em pequenos frascos, as amostras dos polímeros foram dissolvidas em tetrahidrofurano (THF) e posteriormente filtradas em filtros Millipore com abertura de $0,45 \mu \mathrm{m}$. As condições de operação utilizadas foram as seguintes: taxa de bombeamento de $1 \mathrm{ml} / \mathrm{min}$, temperatura de $40{ }^{\circ} \mathrm{C}$ (recomendada quando utilizado o solvente THF) e volume de injeção de $100 \mu \mathrm{L}^{[18,20,23,26]}$.

\subsection{Espectroscopia na região do infravermelho (IV)}

Os espectros das 24 amostras foram obtidos em um espectrômetro FT-IR da marca Perkin Elmer ${ }^{\circledR}$, modelo Spectrum 100. O espectrômetro estava equipado com um acessório de refletância total atenuada (ATR), com cristal de diamante. Este acessório elimina o tradicional método de preparação de amostras pela técnica da pastilha de brometo de potássio, tornando a análise ainda mais rápida ${ }^{[27]}$.

Os espectros foram obtidos a temperatura ambiente, no estado sólido, utilizando o intervalo de $650-4000 \mathrm{~cm}^{-1}$. A resolução utilizada foi de $4 \mathrm{~cm}^{-1}$.

\subsection{Espectroscopia Raman}

Os espectros foram obtidos em um espectrômetro Raman Horiba - Jobin Yvon, modelo XploRA. Não é necessário preparação das amostras, e os espectros foram obtidos em condições ambientais normais. A fonte de luz visível tinha comprimento de onda de $532 \mathrm{~nm}$ e a potência do laser foi ajustada para $50 \%$. Os espectros foram obtidos no intervalo entre $350-3500 \mathrm{~cm}^{-1}$ com resolução de $1,8 \mathrm{~cm}^{-1}$. Os parâmetros utilizados foram sugeridos pelo fabricante.

\section{Resultados e Discussão}

\subsection{Resultados dos rendimentos e aspectos gerais do material produzido}

Nesta seção serão apresentados os rendimentos obtidos nas reações (Tabela 2) e os aspectos gerais dos materiais produzidos em diferentes condições.

Tabela 2. Valores de massa e rendimento obtidos nas reações de polimerização.

\begin{tabular}{|c|c|c|c|c|c|c|}
\hline $\mathrm{N}^{0}$ da Ampola & $\begin{array}{c}\text { Massa dos } \\
\text { Reagentes (g) }\end{array}$ & Tempo (h) & $\begin{array}{c}\text { Temperatura } \\
\left({ }^{\circ} \mathrm{C}\right) \\
\end{array}$ & $\begin{array}{c}\text { Massa obtida após } \\
\text { purificação (g) }\end{array}$ & Rendimento (\%) & $\begin{array}{c}\text { Média do } \\
\text { Rendimento }\end{array}$ \\
\hline 1 & 7,187 & 6 & 110 & 4,381 & 60,96 & 58,56 \\
\hline 2 & 7,185 & 6 & 110 & 4,035 & 56,16 & \\
\hline 3 & 7,186 & 24 & 110 & 6,378 & 88,76 & 86,88 \\
\hline 4 & 7,179 & 24 & 110 & 6,103 & 85,01 & \\
\hline 5 & 7,197 & 72 & 110 & 6,797 & 94,44 & 92,87 \\
\hline 6 & 7,188 & 72 & 110 & 6,563 & 91,30 & \\
\hline 7 & 7,191 & 168 & 110 & 5,644 & 78,49 & 80,06 \\
\hline 8 & 7,191 & 168 & 110 & 5,871 & 81,64 & \\
\hline 9 & 7,183 & 6 & 140 & 4,890 & 68,07 & 69,40 \\
\hline 10 & 7,183 & 6 & 140 & 5,080 & 70,72 & \\
\hline 11 & 7,180 & 24 & 140 & 5,340 & 74,38 & 76,29 \\
\hline 12 & 7,187 & 24 & 140 & 5,620 & 78,20 & \\
\hline 13 & 7,187 & 72 & 140 & 3,674 & 51,18 & 46,14 \\
\hline 14 & 7,188 & 72 & 140 & 2,954 & 41,10 & \\
\hline 15 & 7,188 & 168 & 140 & 0,222 & 3,08 & 3,54 \\
\hline 16 & 7,187 & 168 & 140 & 0,287 & 3,99 & \\
\hline 17 & 7,179 & 6 & 170 & 2,554 & 35,58 & 36,06 \\
\hline 18 & 7,177 & 6 & 170 & 2,623 & 36,55 & \\
\hline 19 & 7,179 & 24 & 170 & 2,143 & 29,85 & 28,27 \\
\hline 20 & 7,176 & 24 & 170 & 1,916 & 26,69 & \\
\hline 21 & 7,180 & 72 & 170 & 0,763 & 10,63 & 11,92 \\
\hline 22 & 7,180 & 72 & 170 & 0,949 & 13,22 & \\
\hline 23 & 7,179 & 168 & 170 & $*$ & $*$ & $*$ \\
\hline 24 & 7,180 & 168 & 170 & $*$ & $*$ & \\
\hline
\end{tabular}

* Não houve precipitação após adição de metanol. 
Analisando a Tabela 2, é possível concluir que, considerando apenas o rendimento, os parâmetros indicados para uma produção em série seriam 72 horas e $110^{\circ} \mathrm{C}$, correspondentes às ampolas 5 e 6 . Utilizando estes valores, o rendimento foi superior a $90 \%$. É possível concluir também que a reação não se processa adequadamente em temperaturas elevadas, visto que o máximo de rendimento obtido a $170{ }^{\circ} \mathrm{C}$ foi de $36,06 \%$. O tempo de reação está diretamente ligado à temperatura utilizada. Em temperaturas menores, melhores resultados são obtidos em tempos maiores (porém, tempos demasiadamente longos podem ocasionar uma diminuição no rendimento - vide reações 7 e 8). O inverso ocorre em altas temperaturas, uma vez que há uma diminuição contínua do rendimento com o aumento do tempo de reação.

Embora ótimos rendimentos sejam altamente desejáveis, outros parâmetros e condições do material produzido são igualmente importantes. A seguir são feitos alguns comentários observados ao longo do procedimento utilizado.

Nas ampolas 1 e $2\left(110^{\circ} \mathrm{C}-6 \mathrm{~h}\right)$, o aspecto do polímero após o término da reação era de um líquido bem viscoso e transparente. Este aspecto se manteve mesmo após o resfriamento. Considerando o ponto de fusão do PLGA, na proporção $80 / 20$, em torno de $140^{\circ} \mathrm{C}$, e dos monômeros em torno de $100^{\circ} \mathrm{C}$, conclui-se que o grau de polimerização dos monômeros foi baixo. Após o procedimento de purificação, $\mathrm{o}$ aspecto pegajoso se manteve, sendo muito difícil de retirar completamente a amostra do béquer. A filtração de ambas as amostras foi rápida - cerca de 15 minutos.

Nas ampolas 3 e $4\left(110{ }^{\circ} \mathrm{C}-24 \mathrm{~h}\right)$, ao término da reação, o material estava no estado sólido e tinha aspecto extremamente duro e levemente esbranquiçado. O tempo de dissolução em clorofórmio foi muito maior que os testes 1 e 2 (cerca de 12 horas), sugerindo massas moleculares maiores. Após a precipitação em metanol, as amostras também apresentaram aspecto pegajoso. Entretanto, ao tirar as amostras do solvente, em poucos segundos o material perdia a característica pegajosa e passava a se assemelhar a uma cola depois de seca. O processo de filtração foi semelhante ao dos testes 1 e 2 , levando cerca de 15 minutos para completar a operação. Um detalhe importante observado nos testes 1-4, é que o polímero não desgrudou da ampola após o término da reação. Foi necessário adicionar a amostra + ampola no clorofórmio, com posterior remoção da ampola após completa dissolução. Após a purificação, o aspecto do material era de um pó branco ligeiramente empedrado. $\mathrm{O}$ mesmo aspecto também foi observado nas reações das ampolas 5-20.

O material das ampolas 5 e $6\left(110^{\circ} \mathrm{C}-72 \mathrm{~h}\right)$ e 7 e $8\left(110^{\circ} \mathrm{C}-168 \mathrm{~h}\right)$ apresentaram características muito semelhantes aos anteriores (3-4). Diferenças foram observadas em relação a cor (os 4 últimos já estavam bastante esbranquiçados), e em relação à aderência do polímero na ampola. Os 4 últimos testes foram separados com muita facilidade da ampola, e foram adicionados diretamente no clorofórmio. O tempo de filtração foi ainda menor que os anteriores (cerca de 5 minutos).

Em relação aos testes da próxima bateria $\left(140^{\circ} \mathrm{C}\right)$, o aspecto das ampolas 9-14 era de um líquido bem viscoso e transparente, assim como nos testes 1 e 2. Entretanto, a solidificação do material ocorreu logo após a retirada das ampolas do banho termostático. Isto ocorreu pelo fato da reação ser conduzida acima do ponto de fusão do polímero na proporção 80/20. Diferenças foram encontradas na etapa de purificação do polímero. O líquido contendo clorofórmio + metanol + polímero dissolvido apresentou aspecto turvo com uma coloração esbranquiçada forte, pelo fato de conter ainda em solução o polímero/monômero não reagido. Mesmo após passar pelo filtro, a solução continuava com o aspecto esbranquiçado. Esta condição tornou o processo de filtração muito mais demorado $(>2 \mathrm{~h})$ e ineficiente, diminuindo o rendimento.

Com o objetivo de aumentar o rendimento destas reações, foi adicionado novamente clorofórmio no líquido já filtrado, e em seguida ele foi reprecipitado para ser filtrado pela segunda vez. O aumento no rendimento foi muito pequeno $(\sim 3 \%)$, não justificando a realização de múltiplas filtrações em todos os testes. Os dois últimos testes desta bateria saíram do banho termostático com uma coloração amarelada, e praticamente não houve precipitação da solução polímero + clorofórmio após adição do metanol, mesmo com o dobro do solvente utilizado $(\sim 500 \mathrm{~mL})$. A partir do teste 13 , foram encontradas dificuldades na manipulação do polímero, uma vez que ele voltou a se fixar nas paredes da ampola.

Por fim, na última bateria de testes (17-24), todos os polímeros apresentaram coloração amarelada, aumentando a intensidade da cor com o aumento do tempo de reação. A diferença na coloração sugere uma possível degradação do material devido à altas temperaturas e tempos de reação. Em relação ao rendimento, este diminuiu conforme o tempo de reação tornava-se maior, chegando a $0 \%$ no último caso.

\subsection{Resultados das análises de cromatografia de permeação em gel (GPC)}

Os resultados dos diferentes tipos de massas moleculares (Mn, Mw e Mz), assim como a média da Mw, a massa dos $10 \%$ das cadeias mais pesadas e o índice de polidispersividade são apresentados na Tabela 3.

Analisando a Tabela 3, é possível observar que os polímeros que apresentaram maiores valores de massas moleculares são os dos testes 5 e o 6 (com média de Mw = 17106 Daltons), seguidos pelos dos testes 3 e 4 (com média de $\mathrm{Mw}=15393$ Daltons). É possível observar também que alguns testes apresentam dois valores de massa molecular, sendo um deles indicado com um asterisco. Isso ocorreu pelo fato do surgimento de dois picos ao longo da eluição, com massas moleculares muito diferentes. Este segundo pico, que apresentou em quase todos os casos, massas moleculares muito próximas do valor da massa dos próprios dímero, pode ter sido gerado por material que não reagiu.

Mesmo considerando às baixíssimas massas moleculares em um cálculo separado, o valor do $\mathrm{M}_{\mathrm{w}}$ obtido está abaixo do esperado quando se realiza o processo de polimerização por aberturas de anéis, partindo dos dímeros já purificados. Este valor se encontra próximo aos 100 kDalton. Como já foi citado anteriormente, a reação é extremamente sensível a algumas impurezas, entre elas a água. Além disso, os dímeros utilizados são extremamente reativos, e embora tenham sido armazenados nas condições recomendadas pelo fabricante (freezer), uma parte pode ter sofrido hidrólise e comprometido o crescimento das cadeias. Novos testes 
Peleias, F. S., Jr., Zeituni, C. A., Rostelato, M. E. C. M., Fechine, G. J. M., Souza, C. D., Mattos, F. R., Moura, E. S., Moura, J. A., Benega, M. A. G., Feher, A., Costa, O. L., \& Rodrigues, B. T.

Tabela 3. Valores das diferentes massas moleculares obtidos por GPC.

\begin{tabular}{|c|c|c|c|c|c|c|}
\hline $\mathbf{N}^{0}$ da Ampola & $\begin{array}{c}M_{n} \\
\text { (Daltons) }\end{array}$ & $\begin{array}{c}M_{w} \\
\text { (Daltons) }\end{array}$ & $\begin{array}{c}\text { Média } M_{w} \\
\text { (Daltons) }\end{array}$ & $\begin{array}{c}\mathrm{M}_{\mathrm{z}} \\
\text { (Daltons) }\end{array}$ & $\begin{array}{c}M_{w} 10 \% \text { maiores } \\
\text { (Daltons) }\end{array}$ & $\mathbf{M}_{\mathbf{w}} / \mathbf{M}_{\mathrm{n}}$ \\
\hline 1 & 4173 & 6517 & 8616 & 8593 & 14119 & 1,561 \\
\hline 2 & 7322 & 10716 & & 13950 & 23565 & 1,463 \\
\hline $1 *$ & 297 & 319 & 288 & 340 & 475 & 1,076 \\
\hline $2 *$ & 233 & 256 & & 281 & 415 & 1,097 \\
\hline 3 & 8917 & 14678 & 15393 & 20910 & 36210 & 1,646 \\
\hline 4 & 9862 & 16108 & & 22916 & 39547 & 1,633 \\
\hline $3 *$ & 231 & 257 & 246 & 283 & 425 & 1,111 \\
\hline $4 *$ & 225 & 235 & & 246 & 332 & 1,046 \\
\hline 5 & 9983 & 16285 & 17106 & 23460 & 40607 & 1,631 \\
\hline 6 & 10771 & 17926 & & 25892 & 44699 & 1,664 \\
\hline $5 *$ & 169 & 211 & 211 & 260 & 421 & 1,251 \\
\hline 7 & 2024 & 6306 & 10760 & 12843 & 21563 & 3,115 \\
\hline 8 & 7169 & 15215 & & 24583 & 42185 & 2,122 \\
\hline $8 *$ & 227 & 254 & 254 & 283 & 419 & 1,121 \\
\hline 9 & 4881 & 8151 & 8650 & 12131 & 20987 & 1,670 \\
\hline 10 & 5351 & 9149 & & 13859 & 24052 & 1,710 \\
\hline $9 *$ & 288 & 312 & 284 & 335 & 474 & 1,083 \\
\hline $10^{*}$ & 236 & 256 & & 278 & 412 & 1,084 \\
\hline 11 & 4741 & 9886 & 10551 & 16098 & 27627 & 2,085 \\
\hline 12 & 5617 & 11216 & & 17745 & 30412 & 1,997 \\
\hline $12 *$ & 244 & 254 & 254 & 264 & 352 & 1,040 \\
\hline 13 & 2111 & 4512 & 4749 & 7257 & 12417 & 2,137 \\
\hline 14 & 2158 & 4986 & & 8301 & 14171 & 2,311 \\
\hline 15 & 1756 & 3819 & 3189 & 6287 & 10737 & 2,175 \\
\hline 16 & 1300 & 2559 & & 4135 & 7085 & 1,968 \\
\hline 17 & 1117 & 2142 & 2136 & 3456 & 5925 & 1,917 \\
\hline 18 & 1146 & 2130 & & 3361 & 5766 & 1,859 \\
\hline 19 & 4126 & 8598 & 5440 & 14410 & 24773 & 2,084 \\
\hline 20 & 1131 & 2283 & & 3991 & 6795 & 2,019 \\
\hline 21 & 3348 & 7351 & 8264 & 12119 & 20701 & 2,132 \\
\hline 22 & 4166 & 9178 & & 15134 & 25845 & 2,203 \\
\hline $21 *$ & 230 & 243 & 242 & 257 & 352 & 1,060 \\
\hline $22 *$ & 228 & 240 & & 254 & 349 & 1,055 \\
\hline 23 & 2950 & 5788 & 5764 & 9502 & 16256 & 1,961 \\
\hline 24 & 2198 & 5741 & & 9669 & 16445 & 2,611 \\
\hline $23 *$ & 273 & 300 & 300 & 328 & 458 & 1,099 \\
\hline
\end{tabular}

*Amostras contendo 2 picos diferentes.

utilizando dímeros preparados no próprio laboratório estão sendo realizados. Dessa maneira, os dímeros seriam usados muito mais rapidamente, minimizando os problemas citados acima. Existem relatos na literatura da substituição do vácuo por uma atmosfera inerte, geralmente nitrogênio. Testes adicionais utilizando um fluxo contínuo de nitrogênio em um reator químico também estão sendo realizados.

Relacionando a Tabela 3 (rendimentos) com a Tabela 4 (massas moleculares) é possível concluir que os testes 5 e 6 , seriam mais indicados para uma produção em série. Ambos apresentaram os maiores valores de rendimento e massas moleculares. Os testes 3 e 4 também apresentaram bons rendimentos, boa manuseabilidade e massas moleculares próximas às maiores obtidas $(5$ e 6$)$. Considerando que o tempo de reação dos testes 3 e 4 (24h) é apenas $1 / 3$ do tempo de reação dos testes 5 e 6 (72h), ele também se mostra uma alternativa, caso seja necessário maiores quantidades do polímero em menos tempo.

\subsection{Espectroscopia na região do infravermelho (IV)}

Os polímeros sintetizados foram analisados estruturalmente através de espectroscopia na região IV. Todos os 24 espectros obtidos apresentaram bandas de absorção na mesma região. A Figura 2 apresenta os espectros dos testes 5 e 6, que são aqueles com interesse prático em uma eventual produção.

As bandas destacadas nos espectros acima são representadas na Tabela 4 com as suas respectivas atribuições.

Analisando a tabela com as respectivas bandas de absorção na região do infravermelho, observa-se que todos os grupos funcionais esperados foram de fato encontrados. As amostras dos copolímeros submetidas à análise de espectroscopia IV não apresentaram bandas de absorção entre 3700 e $3100 \mathrm{~cm}^{-1}$, característica de grupamento $\mathrm{OH}$, indicando ausência de umidade nas mesmas. A Figura 3 representa a estrutura do PLGA esperada após a síntese, facilitando a relação dos grupos mostrados na Tabela 4 com os grupos esperados. 
Tabela 4. Atribuição das bandas de absorção na região do infravermelho ${ }^{[18,28-32]}$.

\begin{tabular}{cc}
\hline Banda de Absorção $\left.\mathbf{( c m}^{-1}\right)$ & Atribuição \\
\hline $3000-2950$ & Estiramento antissimétrico $\mathrm{CH}_{3} \mathrm{e} \mathrm{CH}_{2}$ \\
1750 & Estiramento $\mathrm{C}=\mathrm{O}_{\text {do }} \mathrm{COO}$ \\
1454 & Deformação $\mathrm{CH}_{3}$ e CH \\
$1380-1360$ & Deformação $\mathrm{CH}_{3}$ e CH \\
1180 & Deformação C-O do COO \\
$1130-1040$ & Estiramento C-O do CH-O ou CH $\mathrm{CH}_{2}-\mathrm{O}$ \\
865 & Estiramento C-COO \\
755 & Deformação $\mathrm{CH}$ \\
\hline
\end{tabular}

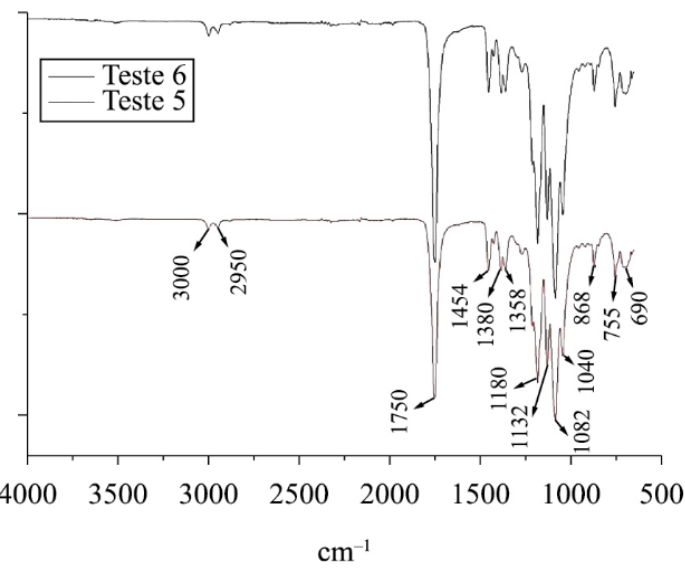

Figura 2. Espectro na região IV do copolímero PLGA 80:20 - Testes 5 e 6.

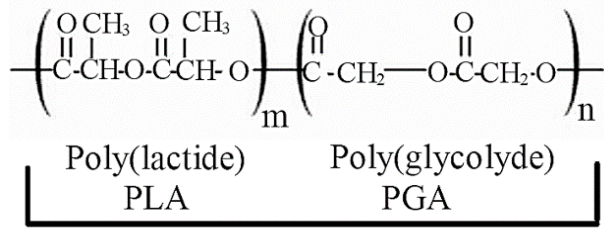

PLGA

Figura 3. Estrutura esperada após síntese do copolímero PLGA ${ }^{[29]}$.
Por último, para fins de comparação, a Figura 4 representa o espectro do copolímero PLGA obtido na literatura.

Comparando os espectros obtidos neste trabalho com o obtido por Hummel ${ }^{[33]}$, nota-se grande similaridade entre eles. Assim, pode-se concluir que os resultados obtidos para as análises de FTIR estão de acordo com a literatura. Observase uma banda entre 3400 e $3600 \mathrm{~cm}^{-1}$ no espectro mostrado na Figura 3, característica do grupo hidroxila, ausente nos espectros encontrados neste trabalho. Essa banda descrita na literatura, talvez pode ser devido à presença de umidade na amostra, de solvente residual, ou da absorção de $(\mathrm{OH})$ do grupo carboxila resultante da hidrólise do monômero ${ }^{[34]}$.

\subsection{Espectroscopia Raman}

Foram escolhidos os dois melhores testes (5-6) para análise por espectroscopia Raman. Como as duas amostras apresentaram resultados idênticos, apenas uma é apresentada na Figura 5.

Assim como na espectroscopia IV, todos os picos esperados foram encontradas. A descrição das bandas encontradas no espectro é descrita na Tabela 5.

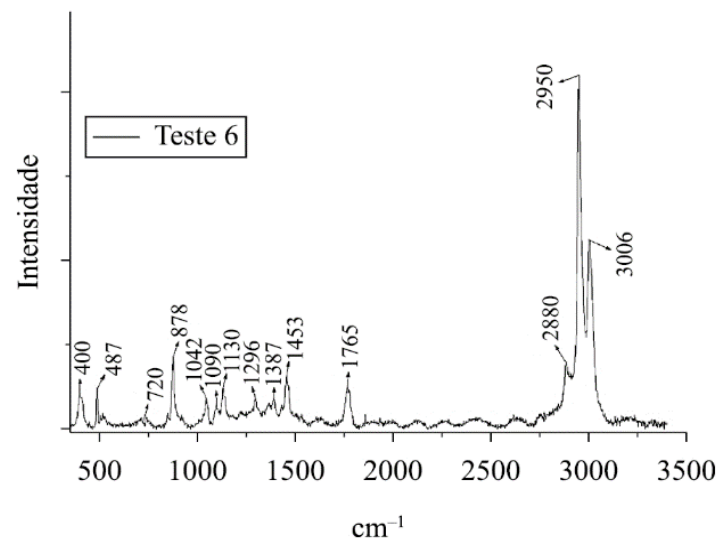

Figura 5. Espectro Raman referente ao PLGA obtido no teste 6.

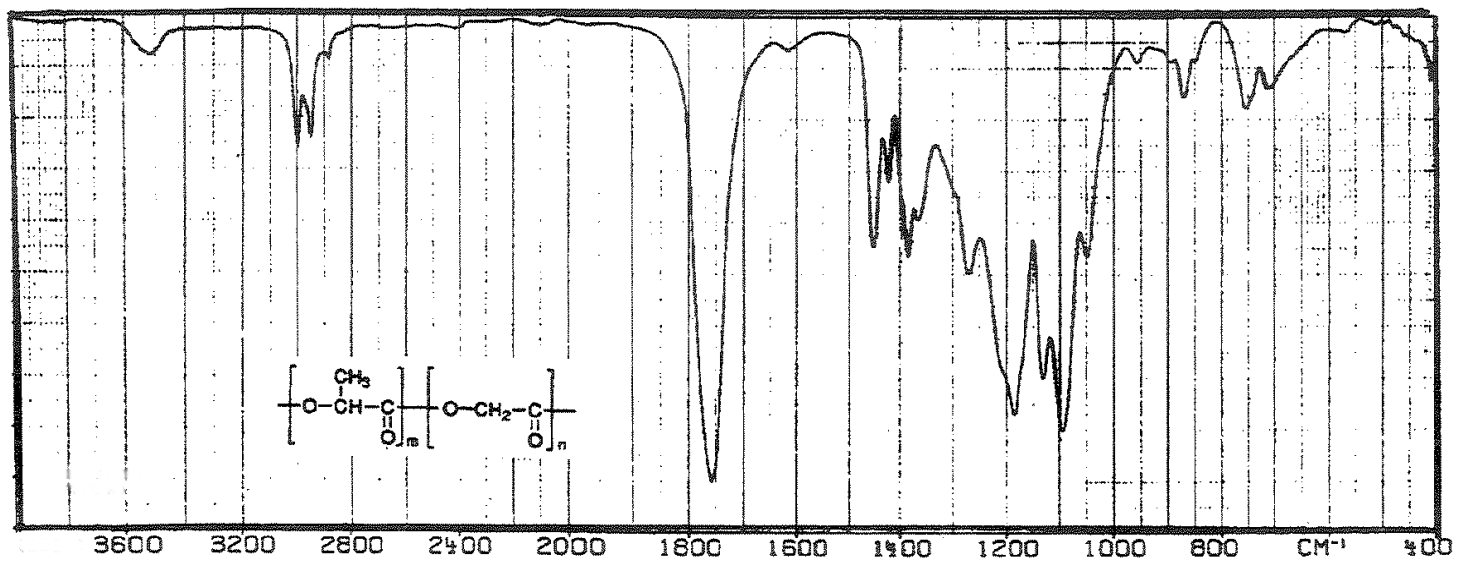

Figura 4. Espectro na região IV referente ao copolímero PLGA obtido na literatura ${ }^{[33]}$. 
Tabela 5. Atribuição das bandas de absorção em espectroscopia $\operatorname{Raman}^{[32,35]}$.

\begin{tabular}{cc}
\hline Banda de Absorção $\left.\mathbf{( c m}^{-1}\right)$ & Atribuição \\
\hline $3006-2950$ & Estiramento antissimétrico $\mathrm{CH}_{3} \mathrm{e} \mathrm{CH}_{2}$ \\
2880 & Estiramento $\mathrm{CH}$ \\
1765 & Estiramento $\mathrm{C}=\mathrm{O}$ \\
1453 & Deformação antissimétrica $\mathrm{CH}_{3}$ \\
1387 & Deformação simétrica $\mathrm{CH}_{3}$ \\
1296 & Deformação $\mathrm{CH}$ \\
1130 & Rotação antissimétrica $\mathrm{CH}_{3}$ \\
1090 & Estiramento simétrico COC \\
1042 & Estiramento C-CH \\
878 & Estiramento C-COO \\
720 & Deformação C=O $(\mathrm{Lactídeo})$ \\
400 & Deformação CCO \\
\hline
\end{tabular}

\section{Conclusões}

Os resultados obtidos demonstram que, através da metodologia proposta para síntese dos polímeros, foi possível determinar os melhores parâmetros de reação (tempo e temperatura) para o PLGA na proporção 80/20. Com uma temperatura de $110^{\circ} \mathrm{C}$ e tempo de reação de $24 \mathrm{~h}$, foi possível obter $86 \%$ de rendimento, e aumento o tempo de reação para $72 \mathrm{~h}$, na mesma faixa de temperatura, o rendimento é superior a $90 \%$. Os resultados obtidos por GPC, também mostram que as maiores massas moleculares foram obtidas nos testes com os maiores valores de rendimento. No entanto, os resultados de massa molecular obtidos são baixos quando comparados com os valores obtidos por outros autores na literatura. Novos testes estão sendo conduzidos, utilizando dímeros preparados no laboratório. Como o tempo entre o preparo e a utilização do dímero seria bem mais curto, problemas envolvendo a hidrólise do material seriam evitados. Testes substituindo o vácuo por uma atmosfera de nitrogênio também estão sendo realizados. Essas duas substituições podem aumentar o valor final da massa molecular do polímero. Em relação à caracterização da estrutura da molécula, as técnicas de espectroscopia IV e Raman confirmaram a estrutura da molécula esperada.

\section{Referências}

1. World Health Organization - WHO. (2012). Cancer. WHO. Recuperado em 12 de julho de 2012, de http://www.who.int/ mediacentre/factsheets/fs297/en/

2. Boyle, P., Levin, B. (Eds.). (2008). World Cancer Report 2008. Lyon: International Agency for Research on Cancer. Recuperado em 12 de março de 2013, de http://www.iarc.fr/ en/publications/pdfs-online/wcr/2008/wcr_2008.pdf

3. International Agency for Research on Cancer-IARC. Globocan 2008. Fast Stats - World. Recuperado em 14 de Julho de 2012, de http://globocan.iarc.fr/Pages/fact_sheets_cancer.aspx

4. Brasil. Ministério da Saúde. Instituto Nacional de Câncer José Alencar Gomes da Silva - INCA. (2011). Estimativa 2012: incidência de câncer no Brasil. Rio de Janeiro: Inca. Recuperado em 12 de março de 2013, de http://portal.saude. sp.gov.br/resources/ses/perfil/gestor/homepage/estimativas-deincidencia-de-cancer-2012/estimativas_incidencia_cancer_2012. pdf
5. Brasil. Ministério da Saúde. Instituto Nacional de Câncer José Alencar Gomes da Silva - INCA. Câncer de próstata. Rio de Janeiro: Inca. Recuperado em 15 de julho de 2010, de http:// www2.inca.gov.br/wps/wcm/connect/tiposdecancer/site/home/ prostata

6. Balter, P. A.; Aguirre, J. F.; Hanson, W. F. (2000). Practical considerations for the calibration of low energy/low activity seeds. In Proceedings of the $22^{\circ}$ Annual EMBS International Conference (pp. 2761-2764). Chicago: IEEE. Recuperado em 12 de março de 2013, de http://ieeexplore.ieee.org/stamp/ stamp.jsp?arnumber $=901433$

7. Li, Z. (2002). Monte Carlo calculations of dosimetry parameters of the urocor prostaseed 125I source. Medical Physics, 29(6), 1029-1034. http://dx.doi.org/10.1118/1.1478559. PMid:12094972.

8. Franca, C. A. S., Vieira, S. L., Bernabe, A. J. S., \& Penna, A. B. R. (2007). The seven-year preliminary results of brachytherapy with Iodine-125 seeds for localized prostate cancer treated at a Brazilian single-center. International Brazilian Journal of Urology, 33(6), 752-762, discussion 762-763. http://dx.doi. org/10.1590/S1677-55382007000600003. PMid:18199343.

9. Gupta, A. P., \& Kumar, V. (2007). New emerging trends in synthetic biodegradable polymers - Polylactide: A critique. European Polymer Journal, 43(10), 4053-4074. http://dx.doi. org/10.1016/j.eurpolymj.2007.06.045.

10. Astete, C. E., \& Sabliov, C. M. (2006). Synthesis and characterization of PLGA nanoparticles. Journal of Biomaterials Science. Polymer Edition, 17(3), 247-289. http://dx.doi. org/10.1163/156856206775997322. PMid:16689015.

11. Oliveira, L. F. (2008). Sintese de poli (ácido lático-co-ácido glicólico) através de policondensação catalisada por resina de troca iônica contendo óxido de estanho como co-catalisador (Dissertação de mestrado). Universidade Federal de Goiás.

12. Moon, S. I., Lee, C.W., Taniguchi, I., Miyamoto, M., \& Kimura, Y. (2011). Melt/solid polycondensation of L-lactic acid: an alternative route to poly(L-lactic acid) with high molecular weight. Polymer, 42(11), 5059-5062. http://dx.doi.org/10.1016/ S0032-3861(00)00889-2.

13. Moon, S.-I., Taniguchi, I., Miyamoto, M., Kimura, Y., \& Lee, C.-W. (2001). Synthesis and properties of high molecular weight poly(L-lactic acid) by melt/solid polycondensation under different reaction conditions. High Performance Polymers, 13(2), 189-196. http://dx.doi.org/10.1088/0954-0083/13/2/317.

14. Nair, L. S., \& Laurencin, C. T. (2007). Biodegradable polymers as biomaterials. Progress in Polymer Science, 32(8-9), 762798. http://dx.doi.org/10.1016/j.progpolymsci.2007.05.017.

15. Carothers, W. H., Dorough, G. L., \& Van Natta, F. J. (1932). Studies of polymerization and ring formation. X. The reversible polymerization of six-membered cyclic esters. Journal of the American Chemical Society, 54(2), 761-772. http://dx.doi. org/10.1021/ja01341a046.

16. Kricheldorf, H. R. (2001). Syntheses and application of polylactides. Chemosphere, 43(1), 49-54. http://dx.doi. org/10.1016/S0045-6535(00)00323-4. PMid:11233824.

17. Stolt, M., \& Södergård, A. (1999). Use of monocarboxylic iron derivatives in the Ring-Opening Polymerization of 1-Lactide. Macromolecules, 32(20), 6412-6417. http://dx.doi.org/10.1021/ ma9902753.

18. Motta, A. C. (2002). Sintese e caracterização do Poli(L-ácido lactic)-PLLA e Poli(L-ácido láctico-co-ácido glicólico)- PGLA e estudo da degradação "in vitro" (Dissertação de mestrado). Universidade Estadual de Campinas. Campinas.

19. Edlund, U., \& Albertsson, A. C. (2003). Polyesters based on diacid monomers. Advanced Drug Delivery Reviews, 55(4), 585-609. http://dx.doi.org/10.1016/S0169-409X(03)00036-X. PMid:12706051. 
20. Zhao-Yang, W., Yao-Ming, Z., Fang, W., \& Jun, W. (2005). Syntheses of poly(lactic acid-co-glycolic acid) serial biodegradable polymer materials via direct melt polycondensation and their characterization. Journal of Applied Polymer Science, 99(1), 244-252. http://dx.doi.org/10.1002/app.22468.

21. Inion. (2012). Sport Medicine-General FAQ. United Kingdom. Recuperado em 20 de julho de 2012, de http://www.inion. com/Products/sportmedicine/en_GB/General_FAQ/\#a_ element_11233216180005731

22. Middleton, J. C., \& Tipton, A. J. (2000). Synthetic biodegradable polymers as orthopedic devices. Biomaterials, 21(23), 23352346. http://dx.doi.org/10.1016/S0142-9612(00)00101-0. PMid:11055281.

23. Zhou, S., Deng, X., Li, X., Jia, W., \& Liu, L. (2004). Synthesis and characterization of biodegradable low molecular weight aliphatic polyesters and their use in protein-delivery systems. Journal of Applied Polymer Science, 91(3), 1848-1856. http:// dx.doi.org/10.1002/app.13385.

24. Grijpma, D. W., Nijenhuiss, A. J., \& Pennings, A. J. (1990). Synthesis and hydrolytic degradation behaviour of high-molecularweight l-lactide and glycolide copolymers. Polymer, 31(11), 2201-2206. http://dx.doi.org/10.1016/0032-3861(90)90096-H.

25. Kiremitçi-Gümusderlelioglu, M., \& Deniz, G. (1999). Synthesis, characterization and in vitro degradation of Poly(D,LLactide)/ Poly(D,L-Lactide-Co-Glycolide). Turkish Journal of Chemistry, 23(2), 153-161. Recuperado em 17 de março de 2013, de http:// journals.tubitak.gov.tr/chem/issues/kim-99-23-2/kim-23-2-698033.pdf

26. Machado, P. F. M. P. B. (2009). Investigação da polimerização via radical livre usando iniciadores trifuncionais (Tese de doutorado). Universidade Estadual de Campinas. Campinas.

27. Perkin Elmer. (2008). Universal ATR polarization accessory for spectrum series FT-IR spectrometers. Waltham: PerkinElmer, Inc. Recuperado em 24 de outubro de 2012, de http://www. perkinelmer.com/CMSResources/Images/44-74753PRD_ UniversalATRPolarizationAccessory.pdf

28. Santos, T. C., Rabelo, D., \& Alves, E. S.(2011). Sintese de nanocompósitos de óxidos de estanho em copolímero estirenodivinilbenzeno para aplicações de interesse industrial. In Anais do $34^{\circ}$ Reunião Anual da Sociedade Brasileira de Química (pp. MAT069). São Paulo: SBQ. Recuperado em 31 de outubro de 2012, de http://sec.sbq.org.br/cdrom/34ra/resumos/T1052-2. pdf

29. Erbetta, C. A. C., Alves, R. J., Resende, J. M., Freitas, R. F. S., \& Sousa, R. G. (2012). Synthesis and Characterization of Poly(D,L-Lactide-co-Glycolide) Copolymer. Journal of Biomaterials and Nanobiotechnology, 3(2), 208-225. http:// dx.doi.org/10.4236/jbnb.2012.32027.

30. Hanafy, A. F., El-Egaky, A. M., Mortada, S. A., \& Molokhia, A. M. (2009). Development of implants for sustained release of 5-fluorouracil using low molecular weight biodegradable polymers. Drug Discoveries \& Therapeutics, 3(6), 287-295. PMid:22495663.

31. Abrams, C. B. (1993). Infrared spectroscopy tutorial and reference. New York: Columbia University/PerkinElmer.

32. Vey, E., Rodger, C., Booth, J., Claybourn, M., Miller, A. F., \& Saiani, A. (2011). Degradation kinetics of poly(lactic-coglycolic) acid block copolymer cast films in phosphate buffer solution as revealed by infrared and Raman spectroscopies. Polymer Degradation \& Stability, 96(10), 1882-1889. http:// dx.doi.org/10.1016/j.polymdegradstab.2011.07.011.

33. Hummel, D. O., Baum, A., Zimmerman, H., \& Solti, A.(2001). Atlas of polymer and plastics analysis: defined polymers (3. ed., Vol. 1). Munique: Carl Hanser Verlag GmbH \& Co.

34. Erbetta, C. D. C., Viegas, C. C. B., Freitas, R. F. S., Sousa, R. G. (2011). Síntese e caracterização térmica e química do copolímero poli(D,L-lactídeo-co-glicolídeo). Polímeros: Ciência e Tecnologia, 21(5), 376-382. http://dx.doi.org/10.1590/ S0104-14282011005000063.

35. Gonçalves, C. M. B., Coutinho, J. A. P., \& Marrucho, I. M. (2010). Optical properties. In R. Auras, L-T. Lim, S. E. M. Selke \& H. Tsuji (Eds.), Poly(lactic acid): synthesis, structures, properties, processing, and applications (pp. 97-112). Hoboken: John Wiley \& Sons.

Enviado: Jun. 04, 2013

Reenviado: Jul. 05, 2014 Aceito: Out. 07, 2014 